Simultaneous measurement of physical parameters using FBGs embedded in unidirectional and bidirectional composite materials

This content has been downloaded from IOPscience. Please scroll down to see the full text.

2016 Smart Mater. Struct. 25015007

(http://iopscience.iop.org/0964-1726/25/1/015007)

View the table of contents for this issue, or go to the journal homepage for more

Download details:

IP Address: 193.137.36.94

This content was downloaded on 06/05/2016 at 12:21

Please note that terms and conditions apply. 


\title{
Simultaneous measurement of physical parameters using FBGs embedded in unidirectional and bidirectional composite materials
}

\author{
Luis Costa $^{1,2}$, Matthieu Gresil ${ }^{3}$ and Orlando Frazão ${ }^{1,2}$ \\ ${ }^{1}$ INESC TEC, Rua do Campo Alegre 687, 4150-179 Porto, Portugal \\ ${ }^{2}$ Dept. of Physics and Astronomy, Faculty of Sciences, University of Porto, Rua do Campo Alegre 687, \\ 4169-007, Porto, Portugal \\ ${ }^{3}$ i-Composites Lab, School of Materials, University of Manchester, 79 Sackville Street, Manchester M1 \\ $3 \mathrm{NJ}$, UK
}

Received 20 July 2015, revised 20 October 2015

Accepted for publication 26 October 2015

Published 18 November 2015

\begin{abstract}
A smart material using fibre Bragg gratings (FBGs) embedded into carbon fibre-reinforced polymer for simultaneous measurement of physical parameters was designed, tested, and validated. Two FBGs were embedded in different sections of the composite sample, one fully unidirectional and the other bidirectional, which produced different sensitivities for each FBG sensor. The composite structure was characterized for strain/temperature and curvature/ temperature measurements. The experimental results were compared with and agreed with finite element simulations.
\end{abstract}

Keywords: optical sensors, fiber Bragg gratings, strain, temperature, SHM, curvature

(Some figures may appear in colour only in the online journal)

\section{Introduction}

Composite materials have been used in many applications in both industry and research. The current applications range from civil engineering to aerospace, given the need to achieve a high strength-to-weight ratio and withstand environmental conditions to overcome previous material-imposed limitations. Due to their broad use, it is relevant to study the addition of sensing capabilities for non-destructive testing and structural health monitoring (SHM) of composite parts [1-3].

Optical fibre sensors stand out due to their sensing capabilities, with minimal influence on the material tensile modulus when embedded parallel to the reinforcement $[4,5]$. In particular, fibre Bragg gratings (FBGs) have been extensively studied and show promising results as embedded sensors for composite materials and structures [6] due to their ease in multiplexing [7] and immunity to electromagnetic interference.

FBGs have been able to monitor materials from manufacturing [8-10] to in situ measurements of strain and temperature [11-13], and are also used for damage detection [14-16] and evaluation of patch repairs [17]. One limitation, however, is the cross-sensitivity of temperature and strain [18]. As such, when the sensors are used in operational environments without controlled temperature, thermal compensation has to be applied. Numerous studies have dealt with simultaneous measurement of temperature and strain, by using either different FBG types [19], superstructured gratings [20], simultaneous measurements of the FBG and Raman distributed temperature sensing [21] or birefringent fibres [22]. Most current solutions, however, are not suitable for real-time in situ SHM applications, where reliability and cost efficiency are crucial.

Other solutions use a pair of similar FBG sensors, changing each sensor's response by embedding them in sections of composites with different surrounding local properties. Examples range from employing a different number of layers [23] to using different surrounding materials for each pair of FBGs [12] or alteration of the surrounding 
material properties through induced damage or different geometry [11].

This work presents a novel case involving local alteration of the material properties by using different surrounding ply orientation for each pair of FBGs. The proposed design has been simulated using ABAQUS-CAE finite element analysis (Dassault Systèmes) to predict the mechanical response of the composite materials prior to tensile and three-point bending tests. A special composite material with the ability to be used as a patch for SHM is demonstrated, and the possibility of simultaneous measurements of curvature and temperature using the same sample is demonstrated and validated.

\section{Sensor design}

This work deals with carbon fibre-reinforced polymer (CFRP) plates with embedded FBG sensors, which are able to simultaneously assess their own temperature and strain. By embedding two FBG sensors in sections of the plate with different local material properties, the temperature and strain response of each FBG of a pair is altered, which allows simultaneous temperature and strain measurements of the pair.

FBG sensors measure the reflected wavelength shift. The Bragg equation for the FBG's reflected peak wavelength (Bragg wavelength) is given by:

$$
\lambda_{B}=2 n_{\text {eff }} \Lambda
$$

with $n_{\text {eff }}$ being the propagating mode's effective index and $\Lambda$ being the period length of the grating. The wavelength shift is due to variations in the effective modal refractive index of the fibre core, or in the grating period length. These allow for measurements of strain and temperature.

The temperature induced wavelength shift is given by

$$
\Delta \lambda_{B}^{T}=\lambda_{B}(\alpha+\xi) \Delta T
$$

where $\alpha$ is the coefficient of thermal expansion of the fibre material, and $\xi$ is the fibre thermo-optic coefficient. In fact, since the sensor is embedded in a different material, its temperature response will also be influenced by the thermal expansion of the surrounding material, and a stress-optic coefficient produced by the thermal expansion mismatch as [24]

$$
\Delta \lambda_{B}^{T}=\lambda_{B}\left(\left(\alpha_{B}-\alpha_{F}\right)\left(1-p_{c}\right)+\alpha_{F}+\xi\right) \Delta T
$$

with $\alpha_{B}$ referring to the surrounding material's thermal expansion, $\alpha_{F}$ to the optical fibre's thermal expansion, and $p_{c}$ to the stress-optic coefficient.

The longitudinal strain induced shift is given by

$$
\begin{gathered}
\Delta \lambda_{B}^{\varepsilon}=\lambda_{B}\left(1-\rho_{\alpha}\right) \Delta \varepsilon \\
\rho_{\alpha}=\frac{n^{2}}{2}\left[\rho_{12}-\nu\left(\rho_{11}-\rho_{12}\right)\right]
\end{gathered}
$$

with $\rho_{\alpha}$ being the photoelastic coefficient, where $\rho_{12}$ and $\rho_{11}$ are the components of the fibre-optic strain tensor and $\nu$ is the Poisson's ratio [25].

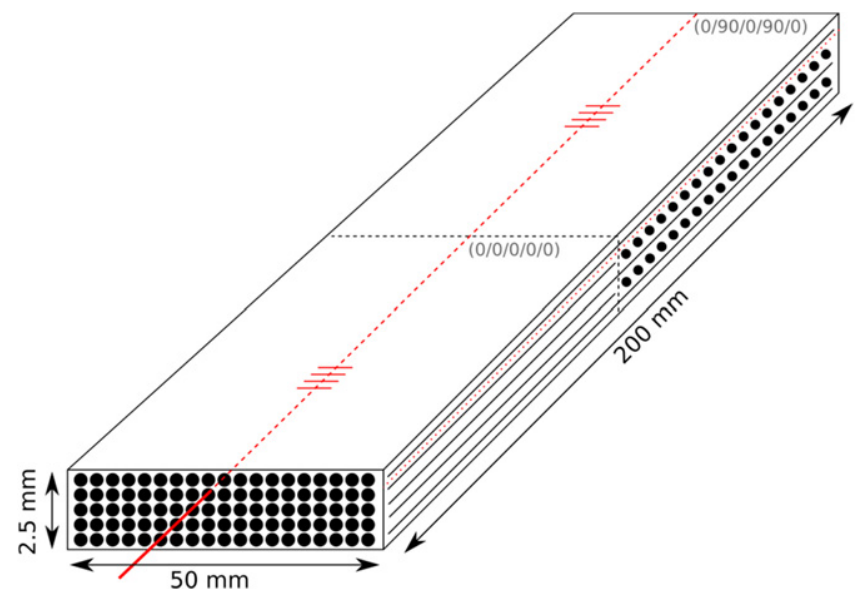

Figure 1. Schematic of the composite sample.

Although the fibre's deformation produces an index shift due to the photoelastic effect, and temperature shifts produce a strain due to thermal expansion of the fibre, the straininduced shift is dominated by the deformation of the grating and the temperature-induced shift is dominated by the thermo-optic effect. The influence of each effect on the produced shift was quantified by Kersey et al [26].

Equations (3) and (4) can be further simplified to

$$
\begin{gathered}
\Delta \lambda_{B}^{T}=\kappa_{T} \Delta T \text { and } \Delta \lambda_{B}^{\varepsilon}=\kappa_{\varepsilon} \Delta \varepsilon \\
\Delta \lambda_{B}=\Delta \lambda_{B}^{\varepsilon}+\Delta \lambda_{B}^{T}
\end{gathered}
$$

with $\quad \kappa_{T}=\lambda_{B}\left(\left(\alpha_{B}-\alpha_{F}\right)\left(1-p_{c}\right)+\alpha_{F}+\xi\right) \quad$ and $\kappa_{\epsilon}=\lambda_{B}\left(1-\rho_{\alpha}\right)$ being the temperature and strain sensitivities for each FBG sensor.

In this case, to solve the cross-sensitivity problem, a pair of FBGs are embedded in a composite plate which consists of a stack of five layers of unidirectional CFRP with two sections, each with a different stack orientation ((0-0-0-0-0) and (0-90-0-90-0)). This changes the FBGs' strain and temperature responses due to the different surrounding material properties, allowing simultaneous measurement of temperature and strain.

\section{Composite plate design}

A rectangular plate of size $300 \mathrm{~mm} \times 50 \mathrm{~mm} \times 2.5 \mathrm{~mm}$ (200 mm gauge length, $50 \mathrm{~mm}$ on each end for gripping), half unidirectional (0-0-0-0-0) and half bidirectional (0-90-0-900 ), with two FBGs embedded along its middle axis, was manufactured by vacuum-assisted resin infusion moulding. The sample schematic can be seen in figure 1 .

Figure 2 shows the finite element model for the longitudinal strain $\left(\varepsilon_{11}\right)$ and the position of the sensors. The simulation was done using $1.5 \times 1.5 \times 0.5$ linear hexahedron type C3D8R elements, applying a tension load ramp to $10 \mathrm{kN}$.

FBGs have a linear response to strain, so sensors should be placed as far from the interface of the two sections and from the edge of the gauge length as possible to ensure linear behaviour. This sample shows a significant increase in 

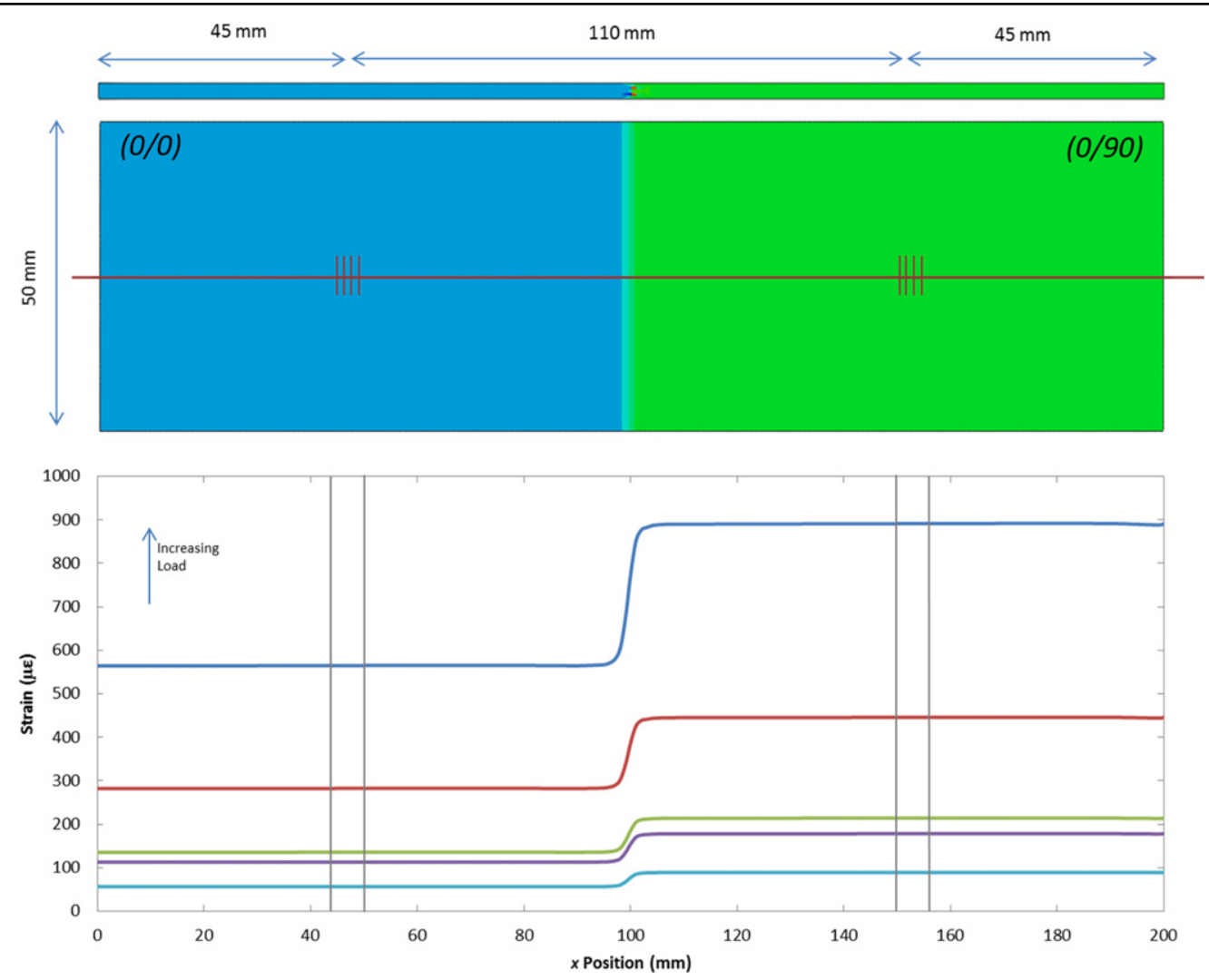

Figure 2. Simulated results of the longitudinal tensile strain versus the $x$ position of the sample. The position of the sensors is clearly stated.

sensitivity on the (0-90-0-90-0) section, due to less longitudinal reinforcement.

The major drawback of this design is the centre interface, which creates a stress concentration and weak point for the composite patch. Due to the nonlinear strain response, the interface may be an interesting zone for further study.

\section{Curvature sensor}

For curvature measurements, using simple trigonometry the following relation is obtained

$$
R=\frac{(L / 2)^{2}+h^{2}}{2 h}
$$

with $R$ being the radius, $h$ the maximum deflection, and $L$ the gauge length of the sample. Since $h$ varies proportionally to the strain at any fixed point in the sample, $h$ can be obtained as a function of the Bragg's wavelength shift (due to strain) in the FBGs. The maximum deflection can be expressed as a function of wavelength as $h\left(\Delta \lambda_{B}^{\varepsilon}\right)=\frac{\Delta \lambda_{B}^{\varepsilon}}{\kappa_{h}}\left(\kappa_{h}\right.$ being the measured sensitivity to the maximum deflection of the sample, measured by the FBG), so the relation between the curvature and wavelength is

$$
C\left(\Delta \lambda_{B}^{\epsilon}\right)=\frac{2 \cdot \frac{\Delta \lambda_{B}^{\varepsilon}}{\kappa_{h}}}{(L / 2)^{2}+\left(\frac{\Delta \lambda_{B}^{\varepsilon}}{\kappa_{h}}\right)^{2}} .
$$

This can be approximated as a linear function, when $h^{2} \ll(L / 2)^{2}$ :

$$
C\left(\Delta \lambda_{B}^{\varepsilon}\right) \cong \frac{2}{\kappa_{h} \cdot(L / 2)^{2}} \cdot \Delta \lambda_{B}^{\varepsilon}=\frac{1}{\kappa_{C}} \Delta \lambda_{B}^{\varepsilon} .
$$

By having two FBG sensors with different sensitivities for both curvature and temperature, their measurements can be easily discriminated when the composite plate is subjected to a three-point bending load.

Figure 3 shows how the strain sensitivity of each FBG relates to its position on the sample when it is subjected to curvature. As such, the position of each of the FBGs is very important to allow simultaneous measurement and eliminate the cross-sensitivity problem. In the sample studied, FBGs were placed $50-55 \mathrm{~mm}$ from the middle of the sample.

Again, the simulation was done using $1.5 \times 1.5 \times 0.5$, linear hexahedron-type C3D8R elements. The surfaces of the sample interact with the three $5 \mathrm{~mm}$-radius bearings, the middle bearing applying the load. The two sections show different strain responses, with the measured compressive strain increasing as it approaches the middle of the sample, where the load is applied.

It is noteworthy that, unlike the tensile strain test, during the three-point bending a strain gradient is observed along the plate sections, and this effect can change the spectral response of the FBGs. 

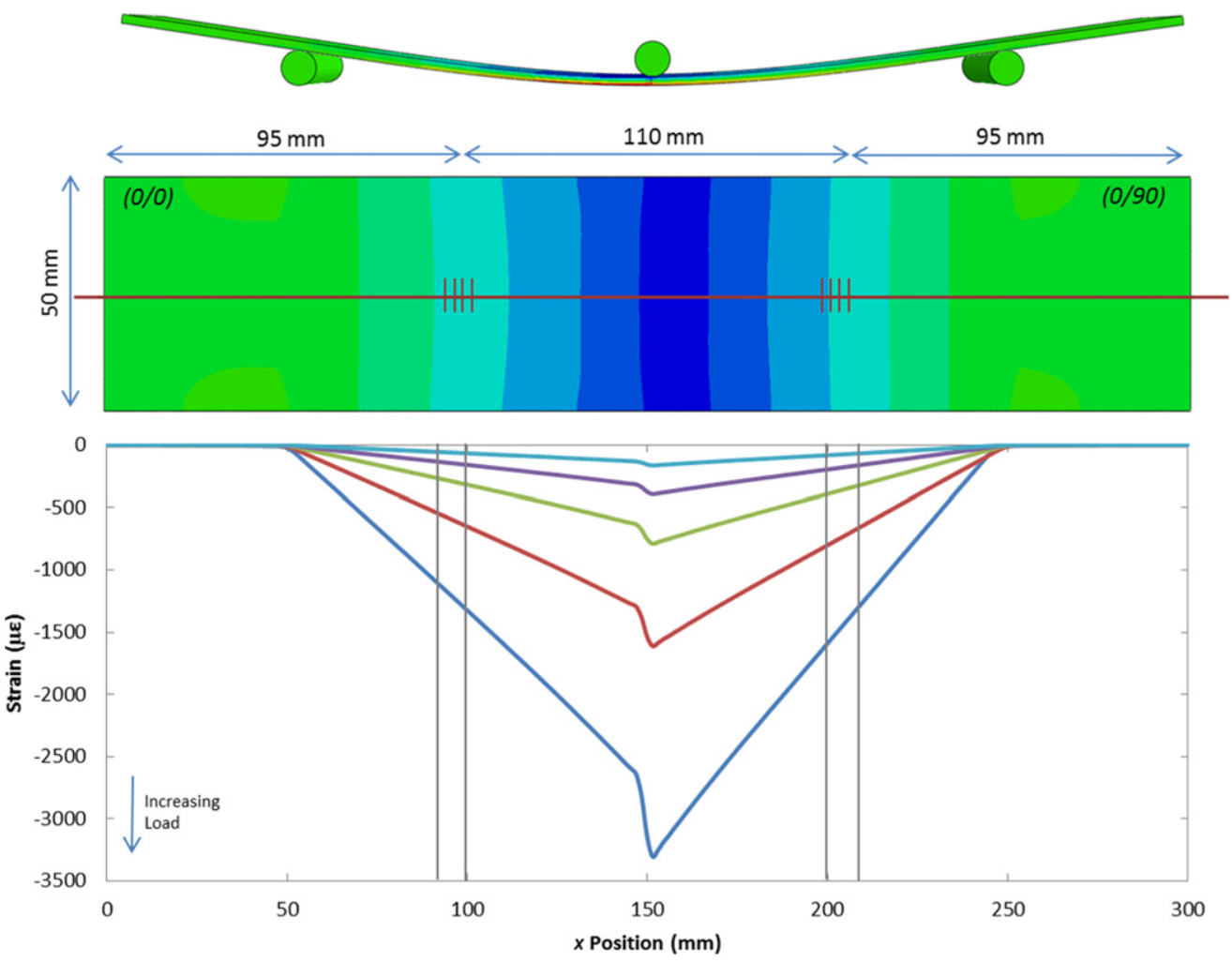

Figure 3. Simulation of the curvature sensor. Sensors are placed on the concave side (measuring compression).

\section{Fabrication of the composite plate}

The sample was manufactured by infusing five layers of SigmaTex unidirectional carbon fibre (T300C, $12 \mathrm{~K}$, $450 \mathrm{gm}^{-2}$ ) with Araldite ${ }^{\circledR}$ LY 564 resin (low-viscosity epoxy resin) and Aradur ${ }^{\circledR} 2954$ hardener (cycloaliphatic polyamine) using the recommended 100:35 weight ratio. The fibre Bragg gratings were $5 \mathrm{~mm}$ SmartFibres SmartFBG (peak reflectivity $>70 \%$ ) imprinted in single mode SMF-28, 9/125 $\mu \mathrm{m}$ fibre, with polyimide recoat at the sensor region.

The layers were cut to the desired shape, with one of the $0^{\circ}$ layers and the $90^{\circ}$ oriented layer being cut in half for later stacking in the aforementioned fashion. For the manufacturing and prior to sensor embedding, all layers except the top layer were stacked. The sensors were then woven through the fabric to ensure their position at the interface of the first and second laminae of the sample and prevent buckling during handling. These were egressed from the side with short polytetrafluoroethylene tubes to protect the egress/ingress region. The final layer was carefully placed on top and the sample was vacuum bagged. The sensors were also egressed from the bag for monitoring the manufacturing process. The cure was performed in a curing oven, using the curing cycle shown in figure 4. The temperature reference was obtained using a Picolog (chromel-alumel) thermocouple. The FBGs measured the temperature and strain changes in the sample during the curing.

The abrupt shifts observed at around $2.5 \mathrm{~h}$ in figure 4 , and the FBG response not matching the thermocouple behavior, are due to the glass transition of the composite resin and the residual strains from the curing process.

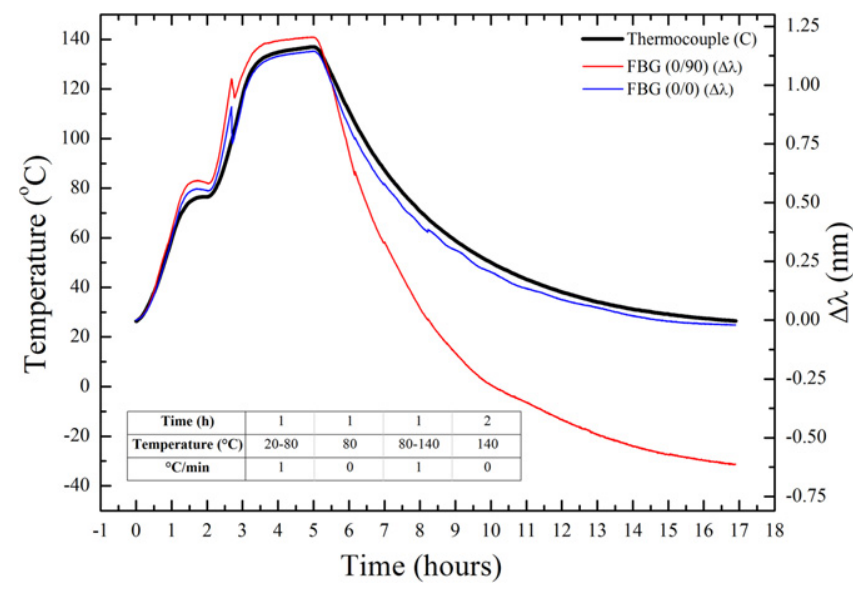

Figure 4. The cycle used for the resin cure, measured through a (chromel-alumel) Picolog thermocouple, and the embedded FBG sensors.

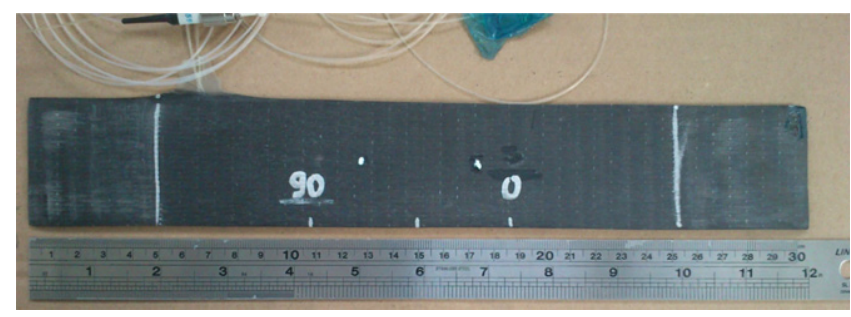

Figure 5. Picture of the sample with a ruler for scale. The bidirectional and unidirectional sections are marked with 90 and 0 , respectively. The dots were used for video extensometer strain measurements. 


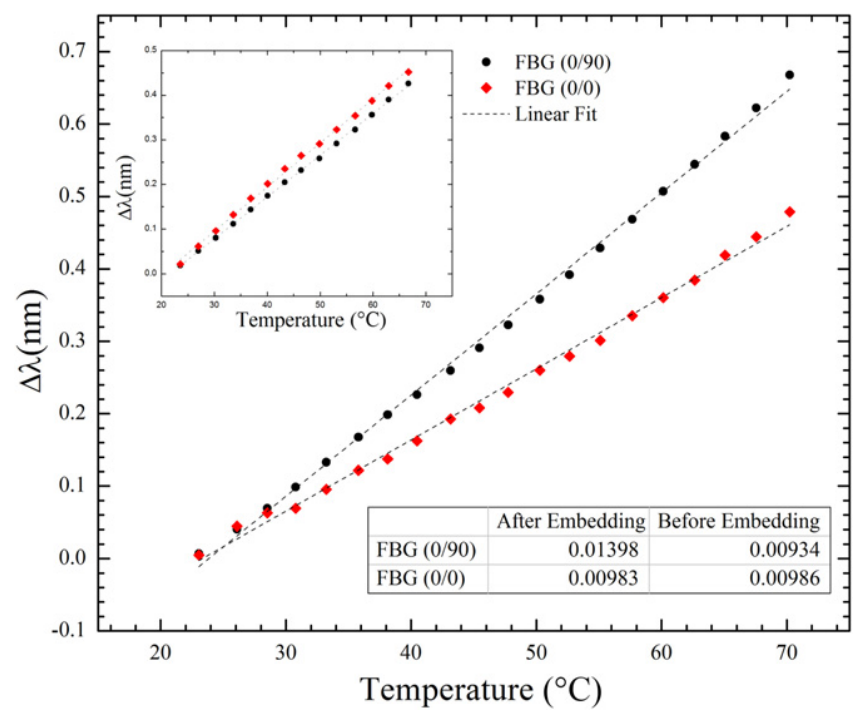

Figure 6. Temperature response of the FBGs before insertion (inset) and after embedding.

After curing, the sample was tailored to the exact length, and any tapering at the edges was removed, as shown in figure 5. Prior to the embedding, both FBGs underwent temperature characterization from 0 to 80 degrees in the same oven used for the cure. After the manufacturing process was complete, the embedded sensors' temperature and strain responses were measured.

\section{Characterization of the composite plate}

All FBG measurements were done using a SmartFibres W4 FBG interrogator (MicronOptics sm125-500 model) with $1 \mathrm{~Hz}$ maximum scan frequency and $1 \mathrm{pm}$ wavelength accuracy, using MicronOptics Enlight software. Reference temperature measurements were done using K-type (chromelalumel) Picolog thermocouples, and a Picolog TC-08 thermocouple data logger, connected to PLW recorder software, with $\pm 0.5^{\circ} \mathrm{C}$ temperature accuracy.

\section{Sensor characterization}

\section{Temperature response}

For the temperature characterization, a temperature sweep from $20^{\circ} \mathrm{C}$ to $80^{\circ} \mathrm{C}$ was performed in an ELKOM vacuum press (with no vacuum applied), without any tensile or compressive loads applied to the plate. The thermocouples were taped to the surface of the sample, closest to the sensors.

Figure 6 shows how the FBG sensors response changed after embedding in the composite plate. The FBG sensors at the (0-90) interface showed an increase in sensitivity to temperature, which may be attributed to stress formed in the manufacturing of the plates at the interface between the $0^{\circ}$ and $90^{\circ}$ laminae. At the (0-0) interface, the sensor showed the least change in its response $(0.3 \%)$, while the sensor at the (0-90) interface showed a great increase in sensitivity

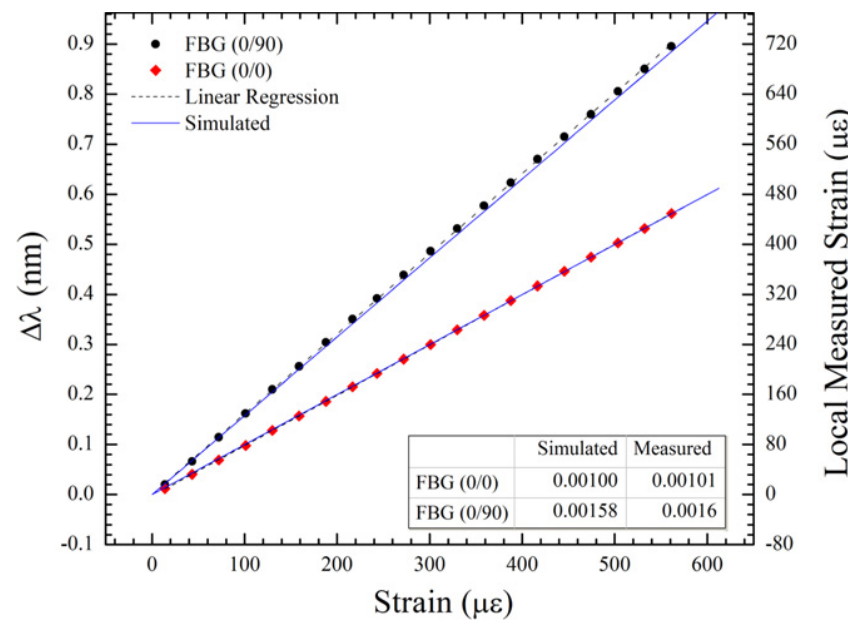

Figure 7. Strain measurements during tensile test and simulated results.

(49.6\%). From this experiment, the thermal sensitivities of the embedded sensors are obtained as $\kappa_{T}^{0 / 0}=9.83 \mathrm{pm}^{\circ} \mathrm{C}^{-1}$ for the sensor at the $(0 / 0)$ interface, with a standard error of $\sigma_{T}^{0 / 0}=0.147 \mathrm{pm}^{\circ} \mathrm{C}^{-1}$, and $\kappa_{T}^{0 / 90}=13.98 \mathrm{pm}^{\circ} \mathrm{C}^{-1}$ for the sensor at $(0 / 90)$, with standard error of $\sigma_{T}^{0 / 90}=0.154 \mathrm{pm}^{\circ} \mathrm{C}^{-1}$.

\section{Strain response}

Strain characterization measurement tests were done using a loading test machine (Instron model 5982), while keeping the plate at constant room temperature $\left(23^{\circ} \mathrm{C}\right)$. Two cycles were done from 0 to $600 \mu \varepsilon$, and the total strain of the sample was measured using an Instron AVE axial strain system. The sample was loaded at $0.20 \mathrm{~mm} \mathrm{~min}^{-1}$. The sample was not subjected to some initial cycles to avoid the potential accommodation effect of FBG sensors to the host material, which can induce some deviations in measurement [27].

Figure 7 shows the simulated and measured strain behavior of the sample. The simulated values were scaled to the (0-0) measurement, which showed the strain sensitivity of the sensors to be $\kappa_{\varepsilon}^{\text {not embedded }} \cong 1.3 \mathrm{pm} \mu \varepsilon^{-1}$.

The simulated strain ratio for the (0-0) and (0-90) section is 1.580 , in excellent agreement with the measured value of 1.584 .

The sensitivities measured for each of the sensors at the $(0-0)$ and (0-90) interfaces are $\kappa_{\varepsilon}^{0 / 0}=1.0 \mathrm{pm} \mu \varepsilon^{-1}$ and $\kappa_{\varepsilon}^{0 / 90}=1.6 \mathrm{pm} \mu \varepsilon^{-1}$, respectively, with standard errors $\sigma_{\varepsilon}^{0 / 0}=6.25 \times 10^{-4} \mathrm{pm} \mu \varepsilon^{-1}$ and $\sigma_{\varepsilon}^{0 / 90}=3.36 \times 10^{-3} \mathrm{pm} \mu \varepsilon^{-1}$.

\section{Curvature response}

The curvature of the composite plate was measured using a three-point bending setup. The sample was loaded at $1 \mathrm{~mm} \mathrm{m^{-1 }}$ on an Instron 5969 fitted with a $10 \mathrm{kN}$ load cell at constant room temperature. Bearings with a $5 \mathrm{~mm}$ radius were placed at the edges of the gauge length, and the sample was loaded along its middle axis. 


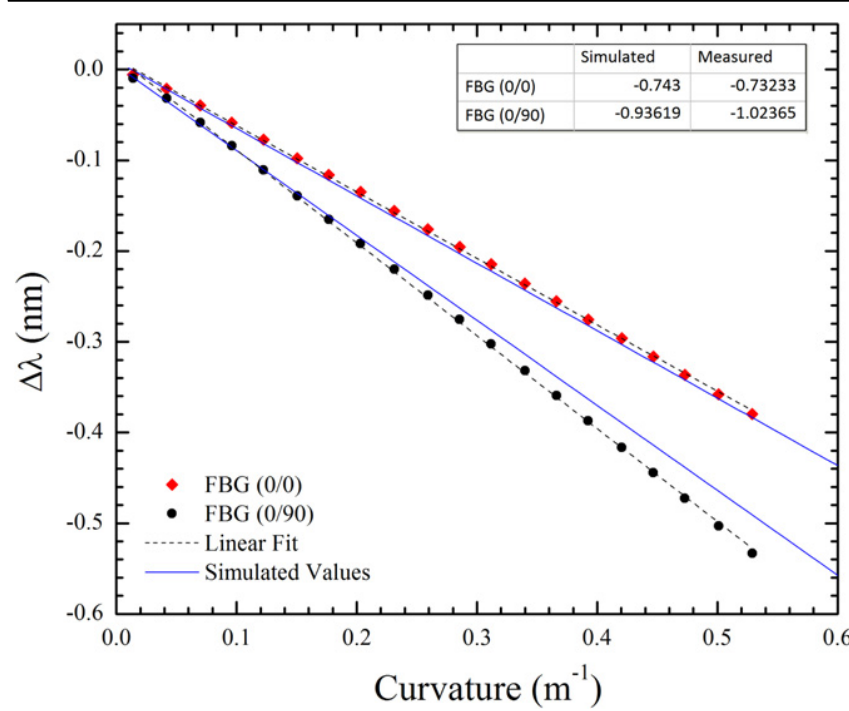

Figure 8. Wavelength shift versus curvature during the three-point bending test, and simulated results.

The sample was loaded from 0 to $3 \mathrm{~mm}$ extension for the curvature measurements, then held and raised up to $5 \mathrm{~mm}$ extension to measure the effects of the strain gradient on the FBG peak, which were found to be negligible for these extension lengths. No relevant spectral deformation was observed for either the quasistatic or the three-point bending tests.

Plotting the wavelength shift as a function of curvature (figure 8) yields linear behavior for small curvatures $\left(h^{2} \ll(L / 2)^{2}\right)$, and the coefficients $\kappa_{C}$ can be obtained as $\kappa_{C}^{0 / 90}=-1024 \mathrm{pm} \mathrm{m}$ and $\kappa_{C}^{0 / 0}=-732 \mathrm{pmm}$, with the standard errors $\sigma_{C}^{0 / 90}=4.74 \mathrm{pm} \mathrm{m}$ and $\sigma_{C}^{0 / 0}=3.56 \mathrm{pm} \mathrm{m}$.

As expected, both sensors measure compressive strain since they are both placed on the concave side of the loaded sample. The simulated ratio of strain for both sections is 1.260 , while the measured ratio of sensitivities is 1.3977 . The discrepancy between the simulated and experimental values may be attributed to displacements of the FBGs during manufacturing in relation to the expected position, which may leave them asymmetrically placed on the sample.

\section{Simultaneous measurement}

The proposed design exhibits different sensitivities to temperature and strain/curvature using similar FBGs. As such, it is possible to obtain a sensor for simultaneous measurements of strain and temperature or of curvature and temperature using this type of composite plate.

Table 1 summarizes the sensitivities for temperature, strain, and curvature measurements.

$$
\left\{\begin{array} { l } 
{ \Delta \lambda _ { B _ { 1 } } = \Delta \lambda _ { B 1 } ^ { \varepsilon } + \Delta \lambda _ { B 1 } ^ { T } } \\
{ \Delta \lambda _ { B _ { 2 } } = \Delta \lambda _ { B 2 } ^ { \varepsilon } + \Delta \lambda _ { B 2 } ^ { T } }
\end{array} \text { or } \left\{\begin{array}{l}
\Delta \lambda_{B_{1}}=\Delta \lambda_{B 1}^{C}+\Delta \lambda_{B 1}^{T} \\
\Delta \lambda_{B_{2}}=\Delta \lambda_{B 2}^{C}+\Delta \lambda_{B 2}^{T}
\end{array}\right.\right.
$$

Table 1. Sensor temperature, strain and curvature sensitivities.

\begin{tabular}{llll}
\hline & $\kappa_{T}\left(\mathrm{pm}^{\circ} \mathrm{C}^{-1}\right)$ & $\kappa_{\varepsilon}\left(\mathrm{pm} \mu \varepsilon^{-1}\right)$ & $\kappa_{C}(\mathrm{pm} \mathrm{m})$ \\
\hline FBG $(0 / 0)$ & 9.83 & 1.0 & -732 \\
FBG $(0 / 90)$ & 13.98 & 1.6 & -1024 \\
\hline
\end{tabular}

Table 2. Sensor temperature, strain and curvature standard errors.

\begin{tabular}{llll}
\hline & $\sigma_{\boldsymbol{T}}\left(\mathrm{pm}^{\circ} \mathrm{C}^{-1}\right)$ & $\sigma_{\varepsilon}\left(\mathrm{pm} \mu \varepsilon^{-1}\right)$ & $\sigma_{C}\left(\mathrm{pm} \mu \varepsilon^{-1}\right)$ \\
\hline FBG (0/0) & 0.147 & $6.25 \times 10^{-4}$ & 3.56 \\
FBG (0/90) & 0.154 & $3.36 \times 10^{-3}$ & 4.74 \\
\hline
\end{tabular}

$$
\begin{gathered}
{\left[\begin{array}{l}
\Delta \lambda_{1} \\
\Delta \lambda_{2}
\end{array}\right]=\left[\begin{array}{ll}
\kappa_{T 1} & \kappa_{\varepsilon 1} \\
\kappa_{T 2} & \kappa_{\varepsilon 2}
\end{array}\right]\left[\begin{array}{l}
\Delta T \\
\Delta \varepsilon
\end{array}\right] \text { or }} \\
{\left[\begin{array}{l}
\Delta \lambda_{1} \\
\Delta \lambda_{2}
\end{array}\right]=\left[\begin{array}{ll}
\kappa_{T 1} & \kappa_{C 1} \\
\kappa_{T 2} & \kappa_{C 2}
\end{array}\right]\left[\begin{array}{c}
\Delta T \\
\Delta C
\end{array}\right]} \\
{\left[\begin{array}{c}
\Delta T \\
\Delta \varepsilon
\end{array}\right]=\frac{1}{D}\left[\begin{array}{cc}
\kappa_{\varepsilon 2} & -\kappa_{\varepsilon 1} \\
-\kappa_{T 2} & \kappa_{T 1}
\end{array}\right]\left[\begin{array}{l}
\Delta \lambda_{1} \\
\Delta \lambda_{2}
\end{array}\right] \text { or }} \\
{\left[\begin{array}{c}
\Delta T \\
\Delta C
\end{array}\right]=\frac{1}{D}\left[\begin{array}{cc}
\kappa_{C 2} & -\kappa_{C 1} \\
-\kappa_{T 2} & \kappa_{T 1}
\end{array}\right]\left[\begin{array}{c}
\Delta \lambda_{1} \\
\Delta \lambda_{2}
\end{array}\right]}
\end{gathered}
$$

$D$ is the determinant of the matrix, $D=\kappa_{\varepsilon 2} \kappa_{T 1}-\kappa_{\varepsilon 1} \kappa_{T 2}$ or $D=\kappa_{C 2} \kappa_{T 1}-\kappa_{C 1} \kappa_{T 2}$ which must be non-zero for possible simultaneous measurement: the greater the determinant, the more accurate the results will be [28]. For greater accuracy in measurement, the difference between the coefficients should be increased.

For these coefficients, the wavelength is measured in pm, giving temperature in degrees Celsius, the strain in $\mu \varepsilon$ and the curvature in $\mathrm{m}^{-1}$ :

$$
\begin{aligned}
& {\left[\begin{array}{l}
\Delta T \\
\Delta \varepsilon
\end{array}\right]=\frac{1}{1.748}\left[\begin{array}{cc}
1.6 & -1.0 \\
-13.98 & 9.83
\end{array}\right]\left[\begin{array}{l}
\Delta \lambda_{1} \\
\Delta \lambda_{2}
\end{array}\right] \text { or }} \\
& {\left[\begin{array}{l}
\Delta T \\
\Delta C
\end{array}\right]=\frac{1}{167.44}\left[\begin{array}{cc}
-1024 & 732 \\
-13.98 & 9.83
\end{array}\right]\left[\begin{array}{l}
\Delta \lambda_{1} \\
\Delta \lambda_{2}
\end{array}\right] .}
\end{aligned}
$$

The respective standard errors are displayed in table 2.

\section{Discussion}

The tested sample design agreed with the previously simulated model for the strain and curvature measurements. The result was a composite patch with embedded fibre Bragg gratings with the ability to be used for SHM purposes.

Prior to infusion and curing, the sample was cut to a size close to the final test piece, allowing minimal handling postcure, and reducing the need to use tools that could endanger the sensor inlets for refining the shape. It is noteworthy that, as represented in the simulated models, the interface at the divide between unidirectional and bidirectional reinforcement 
of the sample produces a clear nonlinear strain and stress behavior which may be interesting to study further.

While this study tested only a CFRP sample, results in other host materials, such as glass-reinforced composites, should be analogous, since the design of the sensor head focuses only on the relative properties of the sensors of each pair, altered by the lamina stack orientation. By embedding the FBG sensors, the temperature and strain/curvature response of each sensor in the pair was altered in relation to the surrounding local material properties of the composite materials. The thermal sensitivity of the FBG embedded at the bidirectional interface increased $49.6 \%$, while the FBG at the unidirectional section remained constant, showing that the FBG sensitivities increase when it is embedded at a bidirectional interface. The sensitivity difference produced by the different material properties of each section enables the development of an analytical model for the discrimination of temperature and strain or curvature and strain, using the Bragg wavelength shift measured by each of the sensors as input.

It may be possible to improve the accuracy of the sensor head by further reducing the longitudinal reinforcement along the bidirectional layer, thus increasing the difference in the strain sensitivities and increasing the determinant of the sensor matrix.

\section{Conclusions}

In summary, a new sensing head based on embedded fibre Bragg gratings on composite plates was designed. The sensing head was experimentally tested and compared against results simulated using finite element methods, with good agreement. The obtained results make it possible to obtain temperature and strain/curvature measurements simultaneously. By embedding the FBG sensors in the part being measured, the optical fibre is protected from damage and moisture and the material can be closely interrogated.

Temperature and strain sensitivities both change when sensors are embedded at a bidirectional interface, while unidirectional embedded sensors keep the thermal sensitivity, resulting in a composite patch that can sense its own alterations in real time, by using a composite material having two distinct sections with different reinforcement orientations.

\section{References}

[1] Diamanti K and Soutis C 2010 Structural health monitoring techniques for aircraft composite structures Prog. Aerosp. Sci. 46 342-52

[2] Gresil M and Giurgiutiu V 2014 Prediction of attenuated guided waves propagation in carbon fiber composites using Rayleigh damping model J. Intell. Mater. Syst. Struct. 26 2151-69

[3] Gresil M, Yu L, Giurgiutiu V and Sutton M 2012 Predictive modeling of electromechanical impedance spectroscopy for composite materials Struct. Health Monit. 11671
[4] Jensen D W 1995 Integrity of composite structures with embedded optical fibers Fiber Optic Smart Structures ed E Udd (New York: Wiley) pp 109-19

[5] Shivakumar K and Emmanwori L 2004 Mechanics of failure of composite laminates with an embedded fiber optic sensor J. Compos. Mater. 38 669-80

[6] Kuang K S C, Kenny R, Whelan M P, Cantwell W J and Chalker P R 2001 Embedded fibre Bragg grating sensors in advanced composite materials Compos. Sci. Technol. 61 1379-87

[7] Jin W 1999 Multiplexed FBG sensors and their applications Proc. SPIE 3897 468-79

[8] Kang H-K, Kang D-H, Bang H-J, Hong C-S and Kim C-G 2002 Cure monitoring of composite laminates using fiber optic sensors Smart Mater. Struct. 11 279-87

[9] Leng J and Asundi A 2003 Structural health monitoring of smart composite materials by using EFPI and FBG sensors Sensors Actuators A 103 330-40

[10] Balvers J M 2014 In situ strain \& cure monitoring in liquid composite moulding by fibre Bragg grating sensors $P h D$ Thesis TU Delft

[11] Rodriguez-Cobo L, Marques A T, López-Higuera J M, Santos J L and Frazão O 2013 New design for temperaturestrain discrimination using fiber Bragg gratings embedded in laminated composites Smart Mater. Struct. 22105011

[12] Ferreira M S, Vieira J, Frias C and Frazão O 2011 Simultaneous measurement of strain and temperature using fiber Bragg grating sensors embedded in hybrid composite laminates Meas. Sci. Technol. 22045206

[13] Doyle C, Martin A, Liu T, Wu M, Hayes S, Crosby P A, Powell G R, Brooks D and Fernando G F 1998 In-situ process and condition monitoring of advanced fibrereinforced composite materials using optical fibre sensors Smart Mater. Struct. 7145

[14] Perez I M, Cui H and Udd E 2001 Acoustic emission detection using fiber Bragg gratings Proc. SPIE 4328 209-15

[15] Propst A, Peters K, Zikry M A, Schultz S, Kunzler W, Zhu Z, Wirthlin M and Selfridge R 2010 Assessment of damage in composite laminates through dynamic, full-spectral interrogation of fiber Bragg grating sensors Smart Mater. Struct. 1915016

[16] Giurgiutiu V, Roman C, Lin B and Frankforter E 2015 Omnidirectional piezo-optical ring sensor for enhanced guided wave structural health monitoring Smart Mater. Struct. 24015008

[17] McKenzie I, Jones R, Marshall I H and Galea S S 2000 Optical fibre sensors for health monitoring of bonded repair systems Compos. Struct. 50 405-16

[18] Kinet D, Mégret P, Goossen K W, Qiu L, Heider D and Caucheteur C 2014 Fiber Bragg grating sensors toward structural health monitoring in composite materials: challenges and solutions Sensors 14 7394-419

[19] Shu X, Liu Y, Zhao D, Gwandu B, Floreani F, Zhang L and Bennion I 2002 Dependence of temperature and strain coefficients on fiber grating type and its application to simultaneous temperature and strain measurement Opt. Lett. 27 701-3

[20] Guan B-O, Tam H-Y, Tao X-M and Dong X-Y 2000 Simultaneous strain and temperature measurement using a superstructure fiber Bragg grating IEEE Photonics Technol. Lett. 12 675-7

[21] Peng F, Ran Z, Rao Y and Liu Y 2012 A hybrid Raman/FBG sensing system for simultaneous measurement of temperature and strain Proc. SPIE 8421 8421A6

[22] Urbanczyk W, Chmielewska E and Bock W J 2001 Measurements of temperature and strain sensitivities of a two-mode Bragg grating imprinted in a bow-tie fibre Meas. Sci. Technol. 12800 
[23] Frazão O, Oliveira R and Dias I 2009 A simple smart composite using fiber Bragg grating sensors for strain and temperature discrimination Microw. Opt. Technol. Lett. 51 235-9

[24] Mora J, Diez A, Cruz J L and Andres M V 2000

Magnetostrictive sensor interrogated by fiber gratings for dccurrent and temperature discrimination IEEE Photonics Technol. Lett. 12 1680-2

[25] Rao Y-J 1997 In-fibre Bragg grating sensors Meas. Sci. Technol. 8355
[26] Kersey A D, Davis M A, Patrick H J, LeBlanc M, Koo K P, Askins C G, Putnam M A and Friebele E J 1997 Fiber grating sensors J. Light. Technol. 15 1442-62

[27] Pereira G, Frias C, Faria H, Frazão O and Marques A T 2013 On the improvement of strain measurements with FBG sensors embedded in unidirectional composites Polym. Test. 32 99-105

[28] Jin W 1997 Simultaneous measurement of strain and temperature: error analysis Opt. Eng., Bellingham 36598 